\title{
PERSPECTIVA DE LOS ESTUDIANTES ACERCA DE LAS PRÁCTICAS DE ENSEÑANZA EN LA UNIVERSIDAD. EL CASO DE LAS CARRERAS DE CIENCIAS DE LA EDUCACIÓN DE LA UNNE
}

\author{
Patricia Malena Delgado (Universidad Nacional del Nordeste)* \\ pmadelgado@yahoo.com.ar
}

Recibido: 29/07/2011 Aceptado: 26/10/2011

\section{Resumen}

En el artículo se presentan resultados de una investigación cuyo objeto de estudio son las percepciones y valoraciones de estudiantes de Ciencias de la Educación de la Universidad Nacional del Nordeste (UNNE) acerca de las prácticas de enseñanza de sus profesores. Se trabajó desde un abordaje cualitativo, con una muestra intencional de doce estudiantes avanzados de la licenciatura y el profesorado utilizando técnicas como las entrevistas en profundidad y los grupos focales.

Los datos obtenidos permitieron realizar una caracterización de las prácticas de enseñanza de los profesores desde la perspectiva de los alumnos, e interpretar en sus discursos los significados atribuidos al conocimiento, la enseñanza y el aprendizaje. Como argumento central, se sostiene que las percepciones y valoraciones de los estudiantes se modifican a lo largo de la carrera, en un proceso de formación que va configurando el modo en que significan la experiencia de ser alumno universitario, y junto con ella, los significados atribuidos al conocimiento, la enseñanza y el aprendizaje.

Las prácticas de enseñanza y otras prácticas institucionales, instituyen sentidos para estos objetos de significación, pero cada sujeto hace una apropiación de esos significados, mediada por el tipo de relación con el saber que construye.

\section{Palabras clave}

Percepciones - Enseñanza Universitaria - Formación - Oficio de Alumno - Relación con el saber.

\footnotetext{
* Magíster y Especialista en Docencia Universitaria. Universidad Nacional del Nordeste.
} 


\begin{abstract}
In the article the results of a research are submitted, which purpose of study are the perceptions and valuations of students of the School of Education Sciences of the UNNE over the practices of teaching of its professors. The work was based on a qualitative approach, with an intentional sample of twelve advanced students of the Degree Course and the teaching course using techniques as in-depth interviews and focus groups.

The data gathered allowed to perform a featuring of the teaching practices of the professors from the student's perspective, and construe in its speeches, the meanings allocated to the knowledge, the teaching and the learning. As core argument, it is stated that the perceptions and valuations of the students are modified throughout the career, in a process of formation, that configures the way in which the experience of being a University student and together with it, the meanings allocated to knowledge, teaching and learning. The teaching practices and other institutional practices constitute senses for these objects of meaning, but each person elaborates his own appropriation of those meanings, mediated by the type of relation with the knowledge that it builds.
\end{abstract}

\title{
Key Words
}

Perceptions - University education - Formation - Student Trade - Relation with knowledge.

\section{Introducción}

En el artículo se presentan resultados de una investigación cuyo objeto de estudio son las percepciones y valoraciones de estudiantes de Ciencias de la Educación acerca de las prácticas de enseñanza de sus profesores. Dicha investigación se desarrolló entre los años 2007 y 2009 en la Facultad de Humanidades de la Universidad Nacional del Nordeste (UNNE), y tuvo como objetivo general interpretar esas percepciones y valoraciones, en el marco de las condiciones institucionales en que se desarrolla la enseñanza y la especificidad del campo disciplinar y profesional de Ciencias de la Educación.

Se investigó desde un abordaje metodológico cualitativo, que permitió la emergencia de conceptos y relaciones teóricas a partir del trabajo en terreno y en diálogo con la teoría. Se trabajó con una muestra intencional de doce estudiantes avanzados de la licenciatura y el profesorado, considerando que podían tener una visión general de las prácticas de enseñanza que desarrollan los profesores en los distintos niveles de las carreras. Para el relevamiento de datos, se realizaron entrevistas en profundidad y un taller con certificación institucional, en el cual, a través de la técnica del grupo focal, se retomaron y profundizaron problemáticas emergentes en las entrevistas individuales. La utilización de esta técnica permitió incluir la dimensión social, relacional e interactiva de las percepciones y valoraciones de los sujetos (Parrilla Latas, 1992). 
En el análisis de la información obtenida se avanzó desde un plano descriptivo, caracterizando las prácticas de los profesores en relación a las estrategias de enseñanza y evaluación desarrolladas, hacia una interpretación de los sentidos hegemónicos que estarían sosteniendo esas prácticas. De esa manera, fue tomando forma la tesis central que articula los hallazgos de la investigación, según la cual, las percepciones y valoraciones de los estudiantes se modifican a lo largo de la carrera en un proceso de formación, que va configurando el modo en que significan la experiencia de ser alumno universitario y los significados atribuidos al conocimiento, la enseñanza y el aprendizaje. Las modalidades de enseñanza y otras prácticas institucionales, instituyen sentidos para estos objetos de significación, pero cada sujeto hace una apropiación de ellos mediada por el tipo de relación que construye con el saber. Esta tesis se fundamenta en una serie de premisas teóricas que es necesario explicitar como encuadre de los análisis sucesivos, por lo que se analizará en más detalle su contenido.

\section{Un encuadre teórico para estudiar las percepciones y valoraciones de los estudiantes}

Los seres humanos interpretan el flujo de la experiencia a través de esquemas perceptivos y valorativos, expectativas, intereses y prioridades. Para Pierre Bourdieu (1991), estos esquemas forman parte del "habitus" que cada sujeto porta en tanto actor social, aludiendo a sistemas de disposiciones duraderas y transferibles que generan y regulan prácticas y representaciones, permitiendo que el sujeto opere en la realidad social sin ser del todo consciente de esos saberes y reglas de acción que pone en juego.

Esas estructuras sociales incorporadas a través del proceso de socialización, proveen una ilusión de comprensión inmediata de la experiencia del mundo, excluyendo una interrogación sobre sus propias condiciones de posibilidad. Aunque gozan de cierta estabilidad, no son estructuras rígidas e inmutables, pues permiten adaptarse a la singularidad de las situaciones que los sujetos atraviesan, ya sea a través de pequeñas modificaciones o mediante una reformulación de los sistemas de disposiciones.

La tesis planteada en la investigación se sostiene en estas premisas. Las percepciones y valoraciones de los estudiantes expresan el habitus que portan en tanto actores sociales, y éste se modifica a lo largo de la carrera en un proceso de formación que va configurando el modo en que significan la experiencia de ser alumno universitario. Formación en el sentido que lo plantea Gilles Ferry (1997), como un trayecto o recorrido a través del cual los sujetos adquieren una cierta forma para ejercer un oficio o una profesión.

Un proceso de desarrollo personal que el sujeto emprende y continúa a lo largo de su carrera, y que consiste en un trabajo sobre sí mismo de desestructuración y reestructuración del conocimiento de la realidad. 
El sentido de esa experiencia de ser estudiante en la Universidad no está dado de antemano; se construye a partir de una cultura y en el marco de una situación. Para Philippe Perrenoud (2006), ningún alumno reinventa constantemente el conjunto de valores y esquemas gracias a los cuales da sentido a las situaciones educativas:

Bebe de un legado, es decir, de un habitus, de un capital cultural que lo ayudan a pensar el esfuerzo, el objetivo, las recompensas, los riesgos; que le permiten evaluar lo que le costará mostrar mala disposición ante una tarea escolar o por otro lado lo que puede esperar de su realización. No se está jamás solo en la construcción del sentido. Cada uno se sube a los hombros de las generaciones precedentes y se rodea de sus condiscípulos. En el medio donde se desenvuelven los alumnos se comparte un conjunto de estereotipos sobre el trabajo escolar (Perrenoud, ob.cit.:210).

La construcción del sentido se ancla en esa cultura de los estudiantes pero también se nutre de la cultura familiar. Sus herencias son muy diversas, por lo que el sentido no escapa a las diferencias culturales y a las desigualdades sociales, en cuanto a los recursos que disponen unos y otros para pensar y dominar la experiencia en la Universidad. Se construye también de acuerdo a lo que se vive, en el marco de la relación intelectual y afectiva entre el alumno y el profesor. Se construye en la conversación, en la manera de presentar las cosas, de dar cabida al otro, de negociar el contrato pedagógico. Depende en parte de la cultura común que puedan construir estos actores a propósito del trabajo académico, los saberes, la comunicación o la evaluación.

De acuerdo a estas premisas teóricas y a los propios hallazgos de la investigación, se plantea a modo de hipótesis, que el sentido de la experiencia académica construida por los estudiantes resulta de un proceso de apropiación de dos tipos de aprendizajes:

- los que corresponden al "oficio de ser alumno", consistente en "hacer y mostrar un conjunto de prácticas, actitudes, vínculos y estrategias, que adquieren sentido en la medida que se adaptan y acomodan a lo esperado y valorado por los otros: instituciones, profesores, compañeros" (Leite, 2000:18).

- los propios de un campo disciplinar y profesional específico, en este caso, las Ciencias de la Educación; es decir, la asimilación de un "habitus profesional" en términos de Philippe Perrenoud (1996, citado en Poggi, 2002), un conocimiento experiencial, de carácter subjetivo, personal y situacional, pero a la vez, propio de un grupo o colectivo profesional.

Se trata de dos conjuntos de saberes, habilidades, actitudes y valores, que en parte intersectan y en parte no, en el sentido de que pueden reforzar ciertas concepciones y prácticas o bien contradecirlas. Pero ambos conforman esa 
suerte de "sentido común" que atraviesa la mirada de los estudiantes, aún reconociéndolos como sujetos que realizan un proceso de apropiación particular de estos esquemas de percepción, valoración y acción. Desde ese sentido común y desde la versión personal que cada uno construye, se atribuyen significados al conocimiento, la enseñanza y el aprendizaje.

Determinadas prácticas institucionales, como las modalidades de trabajo con los alumnos en las cátedras, las normativas académicas, el currículum, o la organización de tiempos, espacios y agrupamientos, emiten "mensajes" que los estudiantes aprenden a reconocer para adaptarse a la vida universitaria. El contenido de esos mensajes se vincula a los sentidos hegemónicos que las prácticas recurrentes han instituido: supuestos acerca del modo en que se dan los procesos implicados en la tarea institucional (enseñanza y aprendizaje), los modos de ser y actuar en distintos roles (docente, alumno, autoridades), el valor del conocimiento. La noción de "estilo institucional" precisamente alude a esos aspectos o cualidades de la acción institucional que, por su reiteración, van configurando una serie de rasgos que se presentan como constancias, y generan la impresión de un "orden natural de las cosas" que conforma la cultura institucional (Fernández, 1993).

Más allá de la acomodación o ajuste a estas condiciones y rutinas, los estudiantes asumen posiciones respecto de esos sentidos instituidos; los resignifican, adoptan, confrontan o matizan. Para comprender estas diferencias en las percepciones y valoraciones se apela a la noción de "relación con el saber", entendida como una relación de sentido y de valor entre un individuo (o un grupo) y los procesos o productos del saber. En ella se puede experimentar al saber como una posesión o como un proceso que permite al sujeto, a partir de saberes adquiridos, producir otros nuevos que le permitan pensar, sentir y transformar su realidad (Beillerot, 1998). El tipo de relación con el saber que los estudiantes construyen configura en gran medida cómo significan la experiencia de ser alumno y cómo conciben el aprendizaje, la enseñanza y el contenido puesto en juego en las situaciones educativas.

\section{Explorando sentidos hegemónicos en la institución desde la mirada de los estudiantes}

A partir de una caracterización de las prácticas de enseñanza de los profesores realizada por los estudiantes, se identificaron concepciones de la enseñanza, el conocimiento y el aprendizaje que estarían sosteniendo las modalidades de trabajo más habituales.

Los estudiantes señalaron que en las clases "teóricas" predominaba la exposición del profesor, variando el grado de participación de los alumnos de acuerdo a su estilo, mientras que en los "prácticos" se producía una dinámica más variada, trabajando en forma individual o grupal, con guías de lectura, producción de monografías e informes escritos, o salidas a terreno para realizar 
observaciones y entrevistas. Esta percepción de la teoría y la práctica como ámbitos diferenciados, con un tiempo, un lugar y una modalidad de trabajo específicos, es coherente con las formas habituales de organizar la enseñanza en las cátedras, enmarcadas en normativas institucionales que refuerzan la separación entre clases teóricas y prácticas, y prescriben actividades distintas para los profesores titulares y adjuntos, como responsables de la transmisión del saber, y en una posición jerárquica subordinada, los Jefes de Trabajos Prácticos y Auxiliares docentes a cargo de la parte práctica.

Los alumnos reconocieron también un esquema de trabajo típico o recurrente en las clases, como ilustra la cita:

"Una clase típica es que el profesor presenta el tema, indica la bibliografía, explica cuestiones puntuales y luego el alumno tiene que arreglarse con todo el material: leerlo, entenderlo, hacer apuntes. Eso me parece a mí que es algo típico, que el alumno vaya procesando su propio aprendizaje con respecto a los materiales." (L.).

Se advierte aquí una clara separación de roles: el profesor explica algunos conceptos y relaciones teóricas, da pautas para la lectura de los textos; mientras que el alumno es el responsable de "arreglarse" con el material. Pero, ¿qué significa para él "arreglarse con el material"? ¿Qué tipo de trabajo cognitivo le demanda? Se enfatiza la lectura, la comprensión, el análisis y la síntesis, el buscar aplicarlo a alguna situación práctica. Escuchar al profesor, comprenderlo y tomar apuntes, entender lo que dicen los autores de los textos leídos... Se trata siempre de un saber que se escucha, se interpreta, se comunica, lo que evidencia una enseñanza con un fuerte carácter verbalista, centrada en la transmisión de un conocimiento teórico producido por los autores.

En esta secuencia, la práctica se presenta como un ámbito posterior a la teoría, que hace posible ilustrar los conceptos o utilizarlos en el análisis de situaciones educativas. El alumno debe saber primero la teoría para después ser capaz de interpretar la realidad, cuando es posible el camino inverso, acercarse a la realidad con curiosidad, para desde allí y en diálogo con la teoría, intentar comprenderla.

El contenido de enseñanza es la "teoría", significada como "lo que dice el autor"; concepción de conocimiento que excluye la teorización personal que el estudiante puede realizar en diálogo con los textos, sus profesores o compañeros, y se presenta como disociada de saberes instrumentales para la intervención. Cabe preguntarse, entonces, qué ocurre con aquellos contenidos de otro orden, con los procedimientos, habilidades y destrezas que también componen el perfil profesional del graduado, y que requieren, para ser aprendidos, de otras estrategias de enseñanza.

En la visión de los estudiantes, esos contenidos no se enseñan o se trabajan muy poco. Cuando señalan que en su formación han tenido "mucha teoría 
y poca práctica", están reclamando una mayor formación técnico instrumental, y más espacios de práctica entendida como "estar en situación de, a ver cómo uno hace", en palabras de un alumno. Demandan intervenir activamente en situaciones educativas: enseñando, planificando, elaborando materiales, investigando... Plantean que esas experiencias van más allá de acercarse a la realidad educativa para conocerla y analizarla; aún reconociendo el valor formativo de esas actividades, piden hacer un ensayo de lo que será en el futuro su actividad profesional.

Las prácticas de evaluación tienden a reforzar estas concepciones de conocimiento, enseñanza y aprendizaje. Los estudiantes reconocen como principal criterio de evaluación el manejo de la teoría, es decir, mostrar que se sabe lo que dice el autor, y también, aunque en un segundo plano, ser capaz de analizar situaciones vinculando datos empíricos y teóricos. Asumir una postura personal que toma distancia del texto, no aparece en el discurso de los estudiantes como criterio de evaluación habitual. Sí la figura del analista y la importancia de la lectura.

Respecto de cómo se evalúan los aprendizajes, los estudiantes reconocen una variedad de instrumentos, pero refieren siempre a situaciones de examen. Pareciera que el significado de la evaluación se reduce al de acreditación, práctica institucional responsable de certificar los conocimientos de los alumnos asignándoles una calificación. (Poggi, ob.cit.) Señalan que en muy pocas materias han realizado experiencias de autoevaluación o de co-evaluación, y que no es habitual en las cátedras el desarrollo de dispositivos de evaluación de procesos. Esto contribuye a reforzar una concepción de la evaluación centrada en la medición de resultados, según la cual quien está autorizado a evaluar es el profesor.

Lo que sí reconocen como práctica de los profesores es una suerte de "evaluación informal", que depende del tipo de relación que se establezca con el alumno. Como lo ilustra la cita:

"...los docentes, con algunos alumnos que están más cerca, porque vienen más o hay más contacto, cuando llega el examen final hacen una devolución: cuando vos empezaste eras así y después yo vi esto y esto, pero a mí, en lo personal, muy pocos profes me han dicho eso..." (A.).

Que la evaluación de procesos dependa del tipo de relación con el profesor, evidencia que en general no se plantean en las cátedras dispositivos de seguimiento de los aprendizajes, de un modo intencional y sistemático. De ahí que algunos estudiantes cuestionen la validez del examen como instrumento de evaluación de los aprendizajes, por ser una situación puntual en la que no se ve el proceso formativo que cada uno ha realizado. También por eso diferencian el rendimiento académico, resultado de las calificaciones obtenidas en prácticos, parciales y finales, y los aprendizajes que efectivamente fueron realizando en 
su trayecto de formación. Las notas - dicen ellos - no siempre reflejan estos procesos, dificultades y logros.

En este sentido, algunos estudiantes plantean que los profesores - que encarnan a las instituciones - piensan y proponen la enseñanza basándose en una percepción del alumno como categoría abstracta, como un sujeto estandarizado, no reconocido como una persona concreta que está haciendo la carrera atravesada por una serie de condiciones familiares, económicas o sociales. Así lo expresa un estudiante:

"La facultad en su exigencia no considera tu contexto, si trabajás o no, tu condición familiar; te aísla mucho y te coloca ahí, en la condición de alumno. A la hora de medir el rendimiento eso no aparece considerado, y yo pienso que es algo importante, porque en definitiva está marcando ese rendimiento" (A.).

La perspectiva de los estudiantes permite aproximarse a los sentidos hegemónicos que estarían sosteniendo las modalidades de trabajo recurrentes, pero también reconocer y comprender los diferentes posicionamientos que los sujetos asumen respecto de esos significados, incluyendo matices, adoptando algunas concepciones y tomando distancia o relativizando otras.

Aunque los estudiantes acuerdan, en general, en el modo en que definen la teoría y la práctica, se perciben diferencias en el discurso respecto de la manera vincular estas dos instancias. Para algunos, como la práctica es la instancia donde se aplica la teoría, ésta adquiere sentido y relevancia si es útil al momento de intervenir. El conocimiento debe tener una aplicación práctica en algunas de las actividades para las que habilita el título; se trata de una teoría "subordinada" a la intervención en una actividad profesional. En otros casos se percibe una relación más dialéctica. Las experiencias prácticas son pensadas como situaciones que demandan un trabajo de "traducción" (no de mera "aplicación") de los conocimientos y las técnicas aprendidas; un trabajo creativo, de resignificación o de producción de ideas nuevas.

Respecto de la evaluación, para algunos alumnos priorizar el conocimiento de la teoría implica, en sí mismo, un aprendizaje de tipo memorístico que se limita a reproducir lo que otros piensan o dicen; en cambio para otros, este criterio es válido y no excluye procesos de elaboración personal. Así lo señala un estudiante:

"...a veces discuto con mis compañeros porque muchos plantean: que nos dejen decir a nosotros lo que queremos decir, quiero hablar y decir lo que yo pienso, porque se pide la cuestión conceptual, la teoría en el sentido de que estén precisos los conceptos, pero apropiarse de esos conceptos, creo que eso es válido.." (A.)

De igual modo, frente a las expectativas de los profesores y a las exigencias de acreditación, "acomodarse" puede significar en algunos casos, renunciar al intercambio de opiniones, a la reflexión personal, al cuestionamiento de 
teorías, subordinando la experiencia de aprendizaje a los requerimientos para ser aprobado. En otros casos, se advierte un posicionamiento diferente, más autónomo e interesado en utilizar los márgenes de libertad disponibles. Como lo expresa una alumna:

"...cuando yo sé que no puedo hacer otra cosa, no te queda otra que adaptarte, pero cuando vos sentís que tenés ese espacio de libertad para tratar de cambiar o de hacer algo autónomo, trato en lo posible de usar eso, porque creo que en definitiva, si uno no tiene una postura y no critica nada, y siente que tiene que responder a todo lo que está establecido y no plantea nada nuevo, no tiene sentido el proceso tampoco..." (Ma.).

La posición asumida por el alumno en la relación pedagógica, que implica una relación asimétrica en términos de poder, estaría dependiendo y a la vez configurando un modo particular de vincularse con el saber y de asumir la propia formación. Se trata de una trama de relaciones personales, relaciones de poder y relaciones con el conocimiento, que configura la escena en que se produce el encuentro con el alumno, mediación necesaria entre los sujetos y el saber.

Esa posición se expresa también al definir qué se espera de los profesores. En las entrevistas se advierten diferentes énfasis respecto de la orientación del docente. En algunas, se valora especialmente la figura del profesor que transmite claramente el conocimiento, guía y despeja dudas; mientras que en otras se aprecia más al docente que estimula la libre expresión de los alumnos, el juicio crítico y el pensamiento autónomo, al docente que deja o autoriza un espacio para que el alumno pueda explorar por sí mismo y apropiarse a su modo del conocimiento. Más allá de estas diferencias, en todos los casos se destaca la figura del docente que ayuda a mirar la realidad de otra manera, a cuestionar los preconceptos, a pensarse a sí mismos desde otra perspectiva, y que lo hace a partir de diferentes estilos y estrategias. De ahí la dificultad que plantean para definir la imagen de "un buen profesor", como un modelo ideal. Valoran diferentes rasgos de distintos profesores e identificándose con ellos van conformando una identidad profesional.

\section{La construcción del sentido de la experiencia académica durante el trayecto de formación}

Los sujetos construyen el sentido de su experiencia académica en la Universidad a lo largo de un proceso de maduración, al que todos los estudiantes entrevistados hicieron referencia de algún modo, en expresiones como ésta:

"Cuando yo empecé a venir era: "me voy a la facultad", después llegó un momento que dije: "no soporto la facultad", porque me daba cuenta que no tenía los hábitos que exigía esto. Después fue: "tengo que ir a la facultad, tengo que volver porque es una salida". (A.). 
De acuerdo a sus relatos, en el ingreso a la Universidad las energías están puestas en aprender a desenvolverse en un ámbito extraño. La mayoría de los alumnos caracteriza sus aprendizajes de primer año como superficiales, fragmentados y memorísticos, quizás por esa necesidad imperiosa de adquirir rápidamente un caudal importante de información que aún no puede procesarse. La comprensión deviene luego, a modo de sucesivos insights, a medida que van adquiriendo un lenguaje técnico, conocimientos, hábitos de estudio y habilidades para la comprensión. Así lo relata una alumna:

"Al principio parece que se estudia más de memoria...El alumno no establece relación entre una materia y la otra porque no recuerda los contenidos, el aprendizaje no es significativo. A partir de tercer año uno se asienta un poco más y cambia. Hay actividades que hemos hecho cuyo sentido recién se comprende después, no en el momento" (M.)

La idea de un proceso de maduración que influye en el tipo de aprendizajes realizados y en cómo se significa la experiencia universitaria, remite a la noción de formación y los tiempos que ésta demanda. En este sentido, Eugéne Enriquez (2002) señala:

...no se puede uno formar con profundidad en poco tiempo, porque formarse implica tener tiempo para vivir una experiencia, entenderla, interpretarla, y al mismo tiempo que recoger todos los frutos que ésta produce, desprenderse de ella si es necesario. Los efectos de la experiencia a veces aparecen mucho más tarde (Enriquez, ob.cit.:150).

En este trayecto de formación, ¿qué cambios reconocen en sí mismos los estudiantes?

Por un lado, han aprendido a adaptarse a las exigencias, los tiempos, los estilos de los profesores y los compañeros de estudio. Incorporaron pautas de comportamiento y actitudes necesarias para avanzar regularmente en la carrera. Por otro lado, reconocen haber adquirido un caudal de conocimientos que les permite analizar la realidad educativa y social desde distintas perspectivas disciplinares, articulándolas en una visión holística. Como lo expresan las citas:

"...lo que rescato es el conocimiento que hay acá adentro. Agradezco a mi familia estar en la Universidad, porque es como que me abrió la cabeza... esa mirada holística y multirreferencial que me brinda la carrera yo valoro mucho. Tengo otra mirada a la que tenía antes, que es la que yo veo en chicos que están estudiando en terciario..." (L.).

"...yo en realidad no soy la misma de antes de haber ingresado. Mi forma de pensar, con respecto a la vida cotidiana, con respecto a la docencia. Una de las grandes cosas que aprendí es tratar de mirar desde distintos puntos de vista una problemática... tener un poco más de libertad en la mirada" (L.). 
La construcción de una perspectiva holística y multirreferencial de los fenómenos educativos, que los alumnos han planteado como una característica específica de las Ciencias de la Educación, resulta en buena medida de una formación que pone el énfasis en el conocimiento de teorías y el análisis de situaciones educativas. Aparece una valoración del conocimiento, que tiene un carácter más bien teórico y se vincula a situaciones concretas a través del análisis. Conocimiento como herramienta que permite "abrir la cabeza", "tener una mirada más libre", cuestionar preconceptos... Y es que el trabajo con el conocimiento disciplinar, produce esos efectos en los sujetos. En palabras de Sara Morgenstern (2010):

...es el conocimiento disciplinar el que permite al alumno acceder a los mecanismos causales y a las conexiones relacionales dentro y con otros campos de estudio, lo cual exige sin duda el esfuerzo de aprender paulatinamente un estilo sistemático de razonamiento, que en modo alguno es producto de la experiencia directa. (Morgenstern, ob.cit.:38).

Tal vez por eso, para la mayoría de los alumnos, la Universidad es significada como un mundo nuevo, un espacio de ruptura, de cambio de miradas, un nuevo horizonte de posibilidades. Como lo expresa un estudiante:

“....accedí a un mundo que antes no existía, ahora lo encontré. Para graficarlo así: una vez estaba leyendo en la biblioteca... y en un momento levanto la vista, y veo que estoy rodeado de libros y es para mí... no vengo de una familia donde había muchos libros, ahora sí estoy tratando de ponerlos yo, p.ero uno dice ¿y esto dónde estaba antes? Esa fue la pregunta que me hice, y está acá, no está en otro lado, y estoy yo..." (A.)

Muchos de nuestros estudiantes no tendrían la posibilidad de ingresar a este "mundo nuevo" si no existiera la universidad pública. A pesar de todos los obstáculos que han ido superando para continuar y concluir sus estudios, tuvieron la posibilidad de estudiar y quieren continuar haciéndolo, concientes de que la formación continúa.

El trabajo con el conocimiento tiene múltiples efectos, aunque no sean tan transparentes o visibles. Se aprecian en una duración, se van integrando con otras experiencias; "uno se acuerda de frases, de teorías, pero en realidad creo que lo importante es lo que cristaliza de todo el conocimiento después...", como lo expresa una estudiante. No sólo transforma la mirada de la realidad social y educativa, sino también el conocimiento de sí mismos. En este sentido, los estudiantes han valorado su participación en dispositivos de formación (sobre todo en el quinto nivel del profesorado), que les permitieron reflexionar sobre la formación profesional desde una mirada personal. Advierten asimismo que estos cambios influyeron en la manera de relacionarse con los otros, los compañeros, los profesores. 
Cuando analizan su trayecto de formación, aluden también a un proceso en el que han ido asumiendo posicionamientos respecto de cómo quisieran ser en el futuro, como profesores o licenciados.

"...a medida que va pasando la carrera, te vas formando en una postura frente al futuro rol docente, cómo uno quisiera dar clase, la relación con los alumnos... Ya sea desde las teorías que te pueden llegar a decir hasta la experiencia misma de cada uno" (M.).

"...es como que uno va rescatando pedacitos de cada persona y va tratando de identificarse. Me di cuenta que hay cosas de acá que me sirvieron de muchas profesoras, uno va creando su propio estilo..." (Ma.).

Han ido discriminando rasgos que valoran como positivos de otros que evalúan negativamente. En ese caso también se trata de un aprendizaje, de un "aprender contra", aprender que no es eso lo que cree o lo que quiere.

\section{Conclusiones}

En el trabajo se ha desarrollado la tesis central que articula los hallazgos de la investigación, primero explicitando el encuadre teórico en que se apoya, y luego mostrando cómo articula la trama de conceptos y relaciones teóricas emergentes del trabajo en terreno.

Se ha avanzado en la comprensión de la experiencia académica de los estudiantes universitarios, que van aprendiendo, por un lado, el oficio de ser alumno, adaptándose a los códigos y requerimientos de los profesores y la institución; y por otro, el oficio de ser profesor o licenciado en Ciencias de la Educación, que supone la adquisición de cierto habitus profesional. Como se ha planteado en el trabajo, se trata de dos conjuntos de saberes, habilidades, actitudes y valores, que en parte intersectan y en parte no, en el sentido de que pueden reforzar ciertas concepciones y prácticas o bien contradecirlas. Es el alumno quien, a través de un trabajo de apropiación, va asumiendo posicionamientos, configurando su experiencia académica y definiendo progresivamente una identidad profesional.

La descripción de las modalidades de trabajo más habituales permite plantear, a modo de hipótesis, la vigencia en el plano de las prácticas de concepciones didácticas y epistemológicas con las cuales se confronta o se pone distancia en los discursos, pero que siguen teniendo un poder estructurador de rutinas y de habitus. Así por ejemplo, desde el discurso pedagógico se define a la evaluación integrada al proceso didáctico como un lugar de producción de conocimientos sobre el mismo, valorizándose la evaluación de procesos o formativa; pero la mirada de los estudiantes revela que en las prácticas cotidianas no se desarrollan en las cátedras mecanismos de seguimiento y acompañamiento de los aprendizajes de los alumnos, ni modalidades de autoevaluación o coevaluación. 
De igual modo, respecto del contenido de la formación y la articulación entre teoría y práctica educativas, continuar sosteniendo modalidades organizativas y propuestas didácticas que separan esas instancias o que promueven una aplicación lineal del concepto en una situación que lo ilustre, no favorece trabajar la relación teoría-práctica de un modo dialéctico. Sería necesario revisar la organización de las actividades, la distribución de tiempos, espacios y docentes a cargo de ellas, buscando una mayor integración; y pensar en incluir, en la medida de lo posible, experiencias de práctica profesional (en el sentido expuesto por los alumnos), de acuerdo a los aportes específicos que cada espacio curricular pueda hacer en relación al perfil profesional de los graduados.

En definitiva, los resultados de investigación evidencian aspectos de la formación que sería necesario revisar y reformular, pero también dan cuenta de un proceso de formación en el que los sujetos han aprendido muchas cosas y, sobre todo, se han "transformado". Es importante valorar y dar continuidad a todo ello, consolidando los logros y potenciándolos con otros aspectos que se puedan mejorar.

\section{Referencias bibliográficas}

- Beillerot, J., Blanchard-Laville, C. y Mosconi, N. (1998). Saber y relación con el saber. Buenos Aires: Paidós.

- $\quad$ Bourdieu, P. (1991). El sentido práctico. Madrid: Taurus.

- Enriquez, E. (2002). La institución y las organizaciones en la educación y la formación. Buenos Aires: Ediciones Novedades Educativas.

- Ferry, G. (1997). Pedagogía de la formación. Buenos Aires: Ediciones Novedades Educativas.

- $\quad$ Fernández, L. (1993). Instituciones educativas. Dinámicas institucionales en situaciones críticas. Buenos Aires: Paidós.

- Leite, A. (2000). Representaciones sobre éxito y fracaso académico en estudiantes universitarios. Informe final. Beca de postgrado, categoría perfeccionamiento. Secretaría General de Ciencia y Técnica de la Universidad Nacional del Nordeste (UNNE).

- Perrenoud, P. (2006). El oficio de alumno y el sentido del trabajo escolar. Madrid: Editorial Popular.

- Parrilla Latas, A. (1992). La entrevista en grupo en el contexto de los estudios sobre profesores. En: A. Estebaranz García y V. Sánchez García (comp.): Pensamiento del profesor y desarrollo profesional I. Conocimiento y teorías implícitas. (pp.349-357) Sevilla: Servicio de Publicaciones de la Universidad de Sevilla.

- Poggi, M. (2002). Instituciones y trayectorias escolares. Replantear el sentido común para transformar las prácticas educativas. Buenos Aires: Santillana. 